\title{
In-silico virtual prototyping multilevel modeling system for Cyborgs (CybSim) as a novel approach for current challenges in biosciencies
}

\author{
Manuel Prado-Velasco \\ Department of Graphic Engineering and Multilevel Modeling in Bioengineering Group, University of Seville, Spain, \\ mpradovaus.es
}

\begin{abstract}
There is a lack of Modeling and Simulation software systems in the bioscience arena that give both solutions compliant with current methodologies in drug discovery (pharmaceutic) and precision medicine (healthcare) fields, besides to support the addition of new biological mechanisms under a multilevel and multiformalism perspective, without penalize strongly the model sharing and reusing. A novel modeling and simulation software that tries to fill the previous gap has been designed (CybSim) and it is presented in this work. CybSim is a platform for multilevel modeling of physiological - cybernetic systems, compliant but not limited to Physiologically based, Pharmacokinetic and Pharmacodynamic (PBPK/PK/PD) methodologies. This capability is governed through the Physiological Scope setting value. The main physiological components are mechanistic. The underlying mechanisms may be changed during the model building thanks to the separation between mechanisms and physiological instances. This capability is based on a multi-layer design. A preliminary version of CybSim has been implemented with OpenModelica (v1.14.1). A PBPK semiphysiological model published previously has been built as a case study to demonstrate the feasibility of CybSim. The accuracy of CybSim was verified during preliminary development phases. The two pointed out capabilities of CybSim demanded an object-oriented and acausal equationbased modeling language, able to support classes' redeclaration, connectors' causality, inner/outer scoping control and packages organization. These features are not supported by other modern acausal equation-based modeling languages like the EcosimPro language.
\end{abstract}

Keywords: Cyborgs, Physiological modeling, PBPK, Mechanistic Modeling, acausal equation-based Modeling

\section{Introduction}

The field of modeling and simulation in biosciences is a mature domain that joins efforts from many areas, including biomedical engineering, mathematical biology, and pharmacology. Two big projects that started at the end of 1990's and 2000's may be cited as reference efforts in bioscience modeling. The Physiome initiative was pre- sented in a report from the Commission of Bioengineering in Physiology to the International Union of Physiological Sciences (IUPS) council at the 32nd World Congress in Glasgow (UK) in 1993 (Hunter 2006, Box 1). The Virtual Physiology Human (VPH) project was initiated by the European Commission in 2007, after the publication of the Strategy for a European Physiome (STEP) (Hunter and Viceconti 2009). Both initiatives share as ultimate goal the research and development in computational models, data and tools for a better comprehension of human body under an integrated approach. Many projects defined under the umbrella of Physiome and VPH have pushed the development of a software framework for building mechanistic mathematical multiscale models from cellular to organ levels, with clinical, pharmacological and scientific applications.

A primary objective of VPH - Physiome initiatives was the establishment of model standards and repositories for which the well-known Extensible Markup Language (XML) was selected as a basis approach. Two relevant examples of modeling standards are CellML ${ }^{1}$ and $\mathrm{SBML}^{2}$. CellML was created for the modeling of cell level dynamics, whereas SBML is a modeling language for networks of biological compounds (e.g. metabolic pathways) described using a systemic approach (Systems Biology). SBML and CellML encode models, metadata, and data, which represent the cited spatial scales (levels) of the physiological system to be modelled, in a robust and accurate manner. However, CellML and SBML models must be linked during the numerical integration to simulate the full system. The modeling and simulation tool that perform this task is an Achilles' heel in this process, because it should be compliant with CellML and SBML and other standard modeling languages that represent the remaining physiological levels (tissues, organs, and living system). An interesting example is the Cardiac Physiome Project shown in Hunter and Viceconti (2009, Figure 6). In practice, the integrated framework must manage the interaction among models executed in different tools (Sauro et al. 2004). This approach is limited to interacting models that represent systems with weak coupling. On

\footnotetext{
${ }^{1}$ www.cellml.org

${ }^{2}$ www.sbml.org
} 
the contrary the accuracy and stability of the full model is degraded due to the jacobian deformation.

The increasing complexity of biosciences compels the model standards to evolve. For instance, the SBML v3 standard tries to give response to the requirements of new mathematical methods and physiological methodologies (Keating et al. 2020). This is a second Achilles' heel of XML modeling languages, since mechanisms and approaches are restricted to fulfil those data formalisms and levels.

As a consequence, although the SBML, CellML and others XML based modeling languages are designed to build multiscale and modular models under a reusable context, the two aforementioned process limitations difficult the model reusing.

Due to the population requirements of the pharmacology industry, the Non Linear Mixed Effect (NLME) models have guided the building of models in this field (Bonate 2011). In addition, Pharmacokinetics (PK) and Pharmacodynamics (PD) methodologies have defined during decades the basis of the deterministic block of NLME models due to their simplicity and success in the description of drug distribution and clinical responses. The PK methodology has evolved to the Physiologically based PK approach (PBPK) to consider anatomical and physiological features and to improve the predictive ability of NLME models, which is required to address with DrugDrug interactions (DDI) and special or vulnerable populations, for which clinical trials are not suitable (Jones, Gardner, and Watson 2009). As a consequence, most of the mature commercial software tools on pharmacology industry are based on NLME with PK/PD (NONMEM (2021)), or PBPK/PD (SimCyp (2021), GastroPlus (2021), Open-Systems-Pharmacology (2021)).

The gold standard in Population PK/PD modeling, NONMEM, has driven a different approach to achieve the model reusing and sharing in pharmacological sciences. Despite NONMEM models are built through their ordinary differential equations (ODEs), the wide diffusion of NONMEM, R mathematical software ${ }^{3}$ and SBML, promoted the development of a new model standard, PharmML, with the aim of promoting the model sharing. A PK/PD model developed in PharmML is managed by a compliant software tool that may also import SBML code and convert the final model to NONMEM, R, SymCyp, and other well-known modeling software tools (Bizzotto et al. 2017).

The pharmacology industry has pushed the acceptation and evolution of physiological models in the framework of the Quantitative Systems Pharmacology (QSP), which is an approach to translational medicine that combines computational and experimental methods to the development and use of molecules and biologic drugs at the beginning of 2000's (Azer et al. 2021). Many advances in QSP Modeling are supported by PBPK/PK/PD-based NLME

\footnotetext{
${ }^{3}$ www.r-project.org/
}

approaches with increasing efforts to facilitate the inclusion of new knowledge discovering and modeling strategies from Physiome - VPH projects. However, PharmML does not give a solution for the model sharing and reusing requirement in QSP.

A different strategy to achieve the model reusing comes from modeling languages based on equations' formalisms that do not depend on the algorithmic causality (Roa and Prado 2006, Figure 8). This approach has proved its feasibility in the engineering field with EcosimPro language (EL) (Empresarios Agrupados 2019) and Modelica (Fritzson 2015) as two cutting edge object-oriented (OO) and acausal equation-based modeling language references. EL is a proprietary language implemented in the EcosimPro software tool from Empresarios Agrupados Internacional (EAI), whereas Modelica is a freely available language from the Modelica Association, implemented in many open and proprietary software tools. This modeling approach has been hardly applied in the biosciences arena so far.

Physiolibrary is a library of specialized Modelica components for the building of complex physiological models (Mateják et al. 2014) that tries to give a solution for the lack of adoption of Modelica in biosciences. It emerged from the construction of a large model of human physiology, Physiomodel, which in turn is an extended Modelica version of the integrative human physiological model called HumMod (Hester et al. 2011). The recent study of Ježek et al. (2017) describes a methodology for creating cardiovascular system models with different complexity based on Physiolibrary with the main objective of demonstrating the feasibility of a standardized platform for model reusing. They show an interesting model that considers the complex interactions between cardiac circulation and arterial systems, founded on a hierarchy of subsystems that takes advantage of the encapsulation and acausal equation-based nature of Modelica. However, neither Physiolibrary (Mateják et al. 2014) nor the derived cardiovascular system model (Ježek et al. 2017) offer a modeling and simulation software tool oriented to the specific requirements and challenges in biosciences. For instance, it is not an easy task to adapt any of them for the solution of parameterized population PBPK models that predict the distribution, therapeutic response and potential interactions of a drug, which is a current standard problem in the pharmacology area.

PhysPK is a software tool for modeling and simulation in biosciences implemented with EL that was designed to fill the gap between open and specialized tools in PBPK/PK/PD that offers multilevel model reusing (PradoVelasco 2016). It was created thanks to an agreement between EAI and me (2015 - 2018) in which I (intellectual owner) worked as team leader, designer and main developer. Several studies have shown the feasibility and accuracy of PhysPK to develop population PK and PBPK models, bioequivalence analysis, and even to generate predictive engines for precision medicine (Reig-Lopez et al. 
2020; Gonzalez-Garcia et al. 2017; Prado-Velasco, Borobia, and Carcas-Sansuan 2020). However, the extension from PBPK to other modeling approaches is limited through the change of the input/output role in selected variables during the translation process, what induces numerical problemas in non-desired flow-pressure transients (any PBPK model in PhysPK is a cardiovascular model). The impossibility to associate chemical names to the enumerated values and the difficulty to manage causal blocks are additional limitations.

In addition, neither Physiolab nor PhysPK has the capability to select the physiological mechanisms during the model building.

In summary, to the best of my knowledge, modeling and simulation software systems fail to provide a standardized framework for specialized bioscience areas like pharmacology (Maharao et al. 2020), precision medicine (Polasek, Shakib, and A. Rostami-Hodjegan 2019; Darwich et al. 2017), toxicology (Paini et al. 2019; Bloomingdale et al. 2017), and regulatory decision (Rowland, Lesko, and Rostami-Hodjegan 2015; Shepard et al. 2015), with multilevel and evolutive model reusing and sharing capability.

The goal of this paper is to show a preliminary version of a novel multilevel modeling and simulation software for physiological - cybernetic systems (Cyborgs) based on Modelica and implemented with OpenModelica 1.14.1, called Cyborg Simulator (CybSim). It has been designed for the aforementioned biosciences areas with emphasis in model reusing. CybSim models may be built according to different modeling methodologies, including PBPK/P$\mathrm{K} / \mathrm{PD}$, and the physiological mechanisms can be selected during the model building, what facilites the model evolution. The diffusion of therapies where a machine is linked to the human body, in a temporal (hemodyalizer) or more permanent manner (artificial heart or insulin pump), is considered through the inclusion of a dedicated machines package. The current presence of computational control and logic in almost all therapy machines explains the selection of Cyborgs as system target. CybSim will be under open source license and available for download.

The work is divided in two stages: a brief presentation of the CybSim design (first) and a study case based on the semiphysiological PBPK model of Mangas-Sanjuan et al. (2018) to demonstrate the feasibility of CybSim (second). The model from Mangas-Sanjuan et al. (2018) was implemented in NONMEM and PhysPK (Reig-Lopez et al. 2020). The experience achieved in that study has facilitated a preliminary and succinct comparison of CybSim against PhysPK. The accuracy of the CybSim PBPK model was verified during the previous development stages.

It is noted that a detailed analysis of more complex physiological models exceeds the scope of this paper, what justifies the use of very single mechanisms in the study case.

\section{Methods}

The study is divided in two stages. The CybSim design is briefly presented in the first stage, which includes two steps.

1. General perspective of CybSim. It includes the package organization and some main setting properties.

2. Modeling strategy. The concept of multilayer design is explained and associated to the separation between mechanisms and physiological entities. Some design concepts related to the machine and signals packages are also presented in this context.

The second stage develops a study case based on a semiphysiological PBPK - based model. It comprises two steps.

1. Building of a semi-physiological model that includes intestinal lumen, gut, liver, a systemic plasma compartment (central) and a peripheral compartment. A solid form of parent drug (PD) is administered. This drug is metabolized in a principal metabolite (PM) and secondary metabolite (SM). The model is presented succinctly in the study case Section, although a detailed description is available (Mangas-Sanjuan et al. 2018; Reig-Lopez et al. 2020).

2. The CybSim model was executed under a periodic administration of the PD solid form, first with the original liver mechanisms of the reference model (Mangas-Sanjuan et al. 2018), and second after the modification of the molecular binding mechanism in the liver.

The study case tries to show the feasibility of CybSim to support the change of any underlying mechanism of a physiological instance during the model building.

Other aspects of the CybSim modeling exceed the scope of the paper. The accuracy of the model was verified during the building process.

\section{CybSim design}

\subsection{General properties and architecture}

Some key issues of the CybSim design are presented in this Section. They referred to the preliminary version 0.2 , implemented in OpenModelica 1.14.1. The Figure 1 shows the packages's organization of CybSim. These are grouped as follows:

- Specific units, main properties and simulation modes of CybSim: SIunits, Properties.

- Connectors and partial classes for the main interfaces: Interfaces.

- Packages that manage biological and physiological data: BioData. 


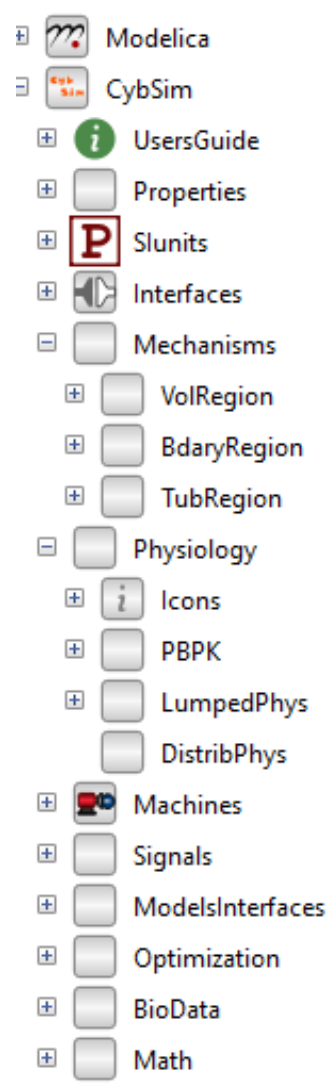

Figure 1. Packages' organization of CybSim v0.2.

- Packages that supports the three CybSim layers: mechanisms, machines and physiological components, and data-driven models: Mechanisms, Machines, Physiology, Signals.

- Templates for model applications at different fields: ModelsInterfaces.

- Auxiliary packages with mathematical methods and Optimization procedures: Math and Optimization.

The main features of CybSim are summarized as follows:

- Physiological multiscope. Physiological subsystems in CybSim may be built according to different modeling methodologies, and thus with different scopes. CybSim v0.2 includes PBPK, LPhys and DPhys. A LPhys (general lumped parameter) model is a generalization of a PBPK model where the blood flow rates result from the cardiovascular circulatory system dynamics. Many times a PBPK, where blood flow rates are set by the modeler, is the right choice for a QSP study, and it has lesser computational requirements. The PBPK physiological scope in CybSim is compliant with the PBPK/PK/PD approach addressed in the Introduction. The DPhys refers to a spatially distributed modeling (not yet madure in
CybSim). The Listing 1 shows the PhysScopeType enumeration type, which controls this feature and it is defined in the properties package.

- Free definition of chemical compounds for models. Chemicals compounds are addressed through a enumeration type (chemicals). The chemicalsDummy type (see Listing 1) is assigned as initial chemicals type, which must be redeclared in the final model, as shown in Listing 9. Several key entities in CybSim are defined in a vectorial (array) mode with chemicals as dimension. The physicochemical properties of the chemical compounds may be defined both manually or from a chemical database.

- Chemical volumes. CybSim v0.2 considers three modes of computing the chemicals volume in solution, governed by the ChemicalvolumeType enumeration (see Listing 1). The NoVolume mode (zero volume) is commonly used for small molecules, whereas the SmallAssolvent considers the true dissolved density of large molecules. This feature requires the discrimination between no large and large molecules. A second enumeration type, mcrChemicals, which must be redeclared in the final model, defines the large molecules, whereas chemicals includes all of them.

- Multilevel modeling. Multilevel is addressed as synonymous of spatial multiscale. This feature is achieved by means of the aggregation (connection) of physiological components at each level, as shown in the conceptual structure of Figure 2 for the physiology subsystem. The same feature is available for the machines and signals subsystem. This is directly derived from the $\mathrm{OO}$ and acausal equationbased property of Modelica and it is available both in textual and graphical model according to the Modelica specification 3.4 (Modelica Association 2017) (MSLv35 has been recently delivered). A key issue here is that the granularity level of different tissues may be different.

- Multilayer architecture. The physical mechanisms that govern the dynamics of any physiological entity are selected during the model building. CybSim achieves this feature through the redeclaration of the inherited mechanisms following the methodology that is explained in the following Section. Conceptually, the physiological layer is defined as a set of physical and mechanistic components that may be defined at different scales (levels) as shown in Figure 2. The mechanism layer is not a physical but an organized set of abstract (partial) components. Although it is not presented in the Figure 2 for the sake of clarity, Machines and Signals may have a multilevel definition and they pertain to Machine and Signals layers, respectively, but they have not a sepa- 


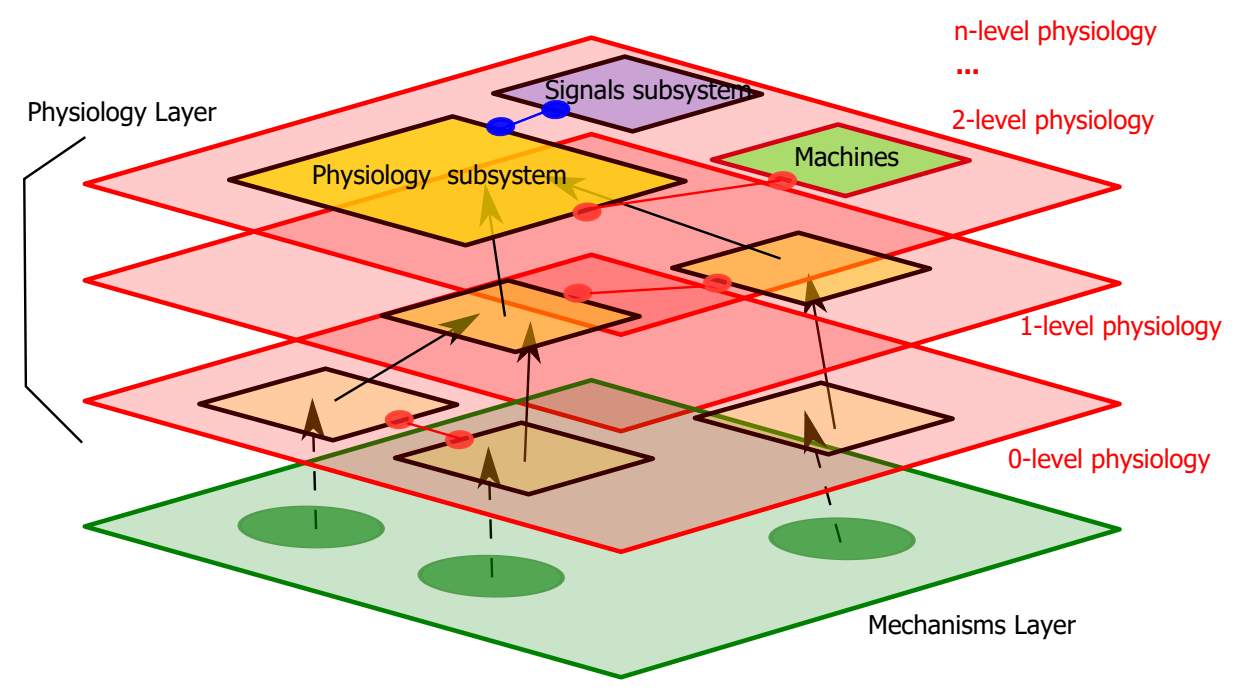

Figure 2. Conceptual structure of a CybSim model that shows the difference between multilevel (multiscale) and multilayer features for physiology entities and their mechanisms (dynamics). Machines and Signals subsystems are presented in the top level of the physiology layer to simplify the structure.

rated mechanistic layer. The fast evolution of the knowledge discovery in the biology field is the reason for this design criterion.

- Genes and Systems Biology. CybSim include metabolic networks based on a Systems Biology approach. This type of metabolic network is defined in the Mechanisms layer using a set of network components compliant with the Systems Biology Graphical Notation (SBGN), which requires the third enumeration type, genes. It is declared initially as the genesDummy type (see Listing 1), which must be redeclared in the final model if Systems Biology Metabolic Networks are used.

- Machines layer. A full machine-physiological system model is considered a cyborg model in CybSim. This generalization of a machine as a cybernetic component is based on the fact that near all machines designed for therapy, support or function enhancement include some type of automatic control systems.

- Signals layer. Data-driven or functional models (Roa and Prado 2006) are designed as Modelica blocks and organized in a package. PD standard models or PK metrics (e.g. Area Under the Curve, AUC) are included here.

A detailed description of the implementation of these features exceed the scope of this paper. However, some relevant issues are clarified in the following paragraphs.

Listing 1. Chemicals definitions and main CybSim scopes

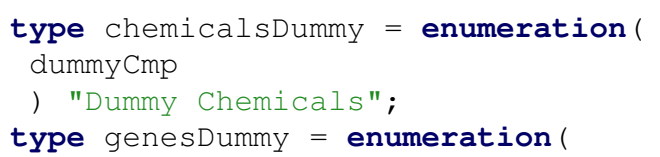

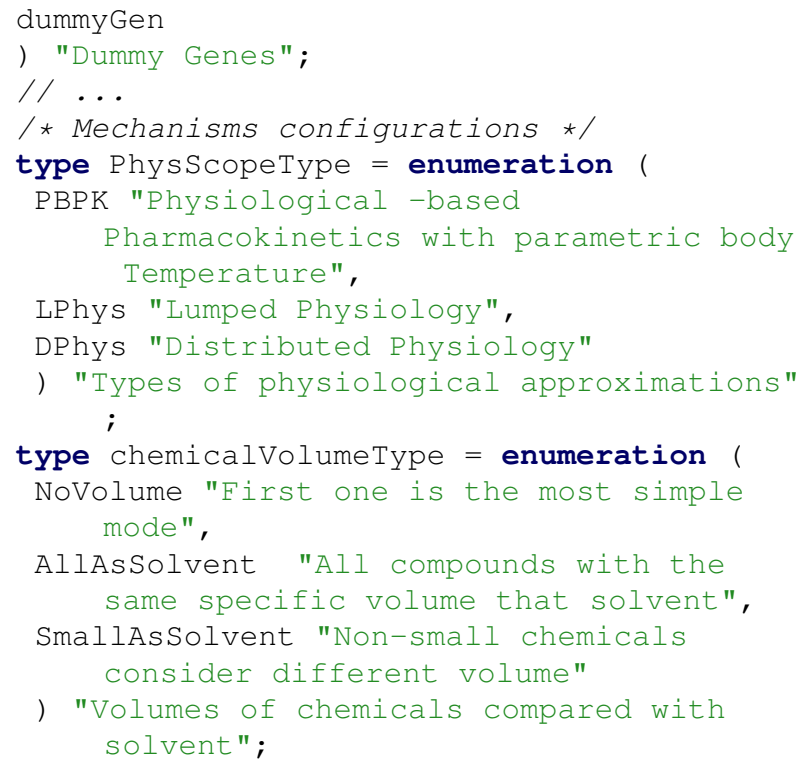

The physiological connectors depend on the scope type as expected, since blood flow rates are user defined with the PBPK scope, whereas they are solved according cardiovascular and circulatory system with the LPhys scope.

The Listing 2 shows the definition of a PBPK input connector as a causal connector that defines chemical and solvent flow rates, and the definition of a LPhys blood connector as an acausal connector that include chemical concentrations as Stream variables to address the reversion of blood flow rates. The later occurs for example in the arterio-venous fistula that connects a dialyzer with a patient. As a consequence, a PBPK model connected to a dialyzer cannot describe some operating conditions that occur with a clotted fistula in some patients. This is not a limitation of CybSim, but of the PBPK modeling approach.

The class bloodConnDummy allows controlling the types of Physiological connectors that may be rede- 
clared in some multiscope CybSim components using the constrainedBy Modelica keyword.

Listing 2. Blood connectors

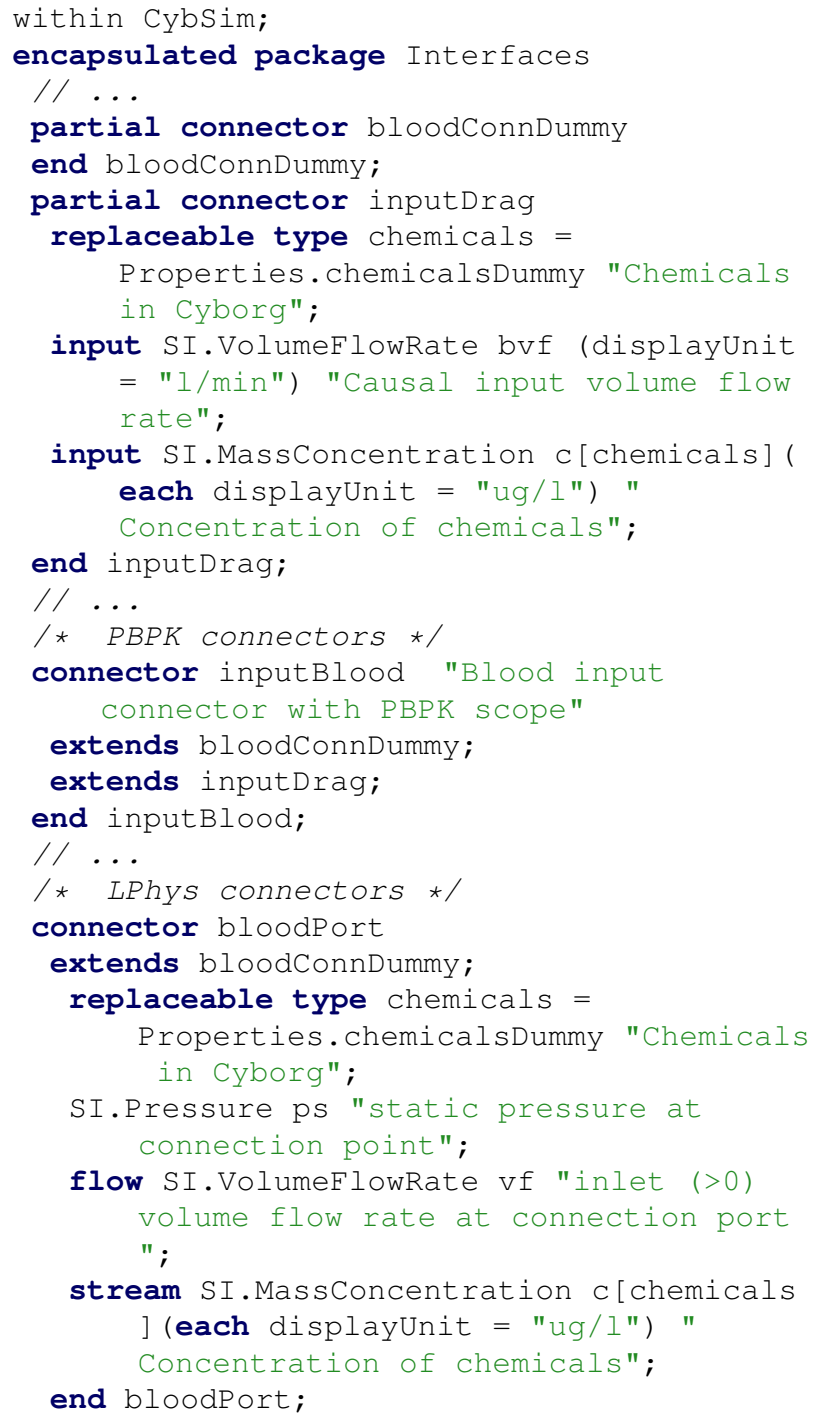

A key declaration in Listing 2 is the replaceable type chemicals that is defined by a shorthand inheritance from the chemicalsDummy enumerated declared in the Listing 1. The type chemicals must be defined in the user's PBPK model according to the chemicals compounds required by the modeler. This feature is not available in other acausal languages as EcosimPro language (EL) (Empresarios Agrupados 2019).

A machine component may connected to any physiological subsystem, and therefore it must adapt their connectors and behaviour to the selected physiological scope. This is achieved thanks to redeclare the machine model and connectors as a function of the physiological scope type, during model building. The Listing 3 presents the technique used for the pharmaceutical drug form machine shown in Figure 3. In this case, the DrugConn connector and the DrugFormScope model that describes the drugForm behaviour may be redeclare, providing that connectors derive from bryConnDummy and machine models de-

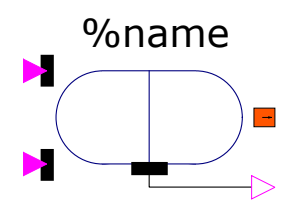

Figure 3. The drug form icon shows two boolean inputs (planned or instantaneous administration setting), one boolean output that informs if a significant drug amount remains to be dissolved, and a physical connector to the liberation lumen.

rive from drugFormDummy. The outer parameter PhysScope has the inner declaration in the final Cyborg model, where it is verified that selected connectors and machine models are compliant with the physiological scope. A deeper analysis exceeds the scope of the paper.

Listing 3. Main Modelica structure of the Ideal drug form machine

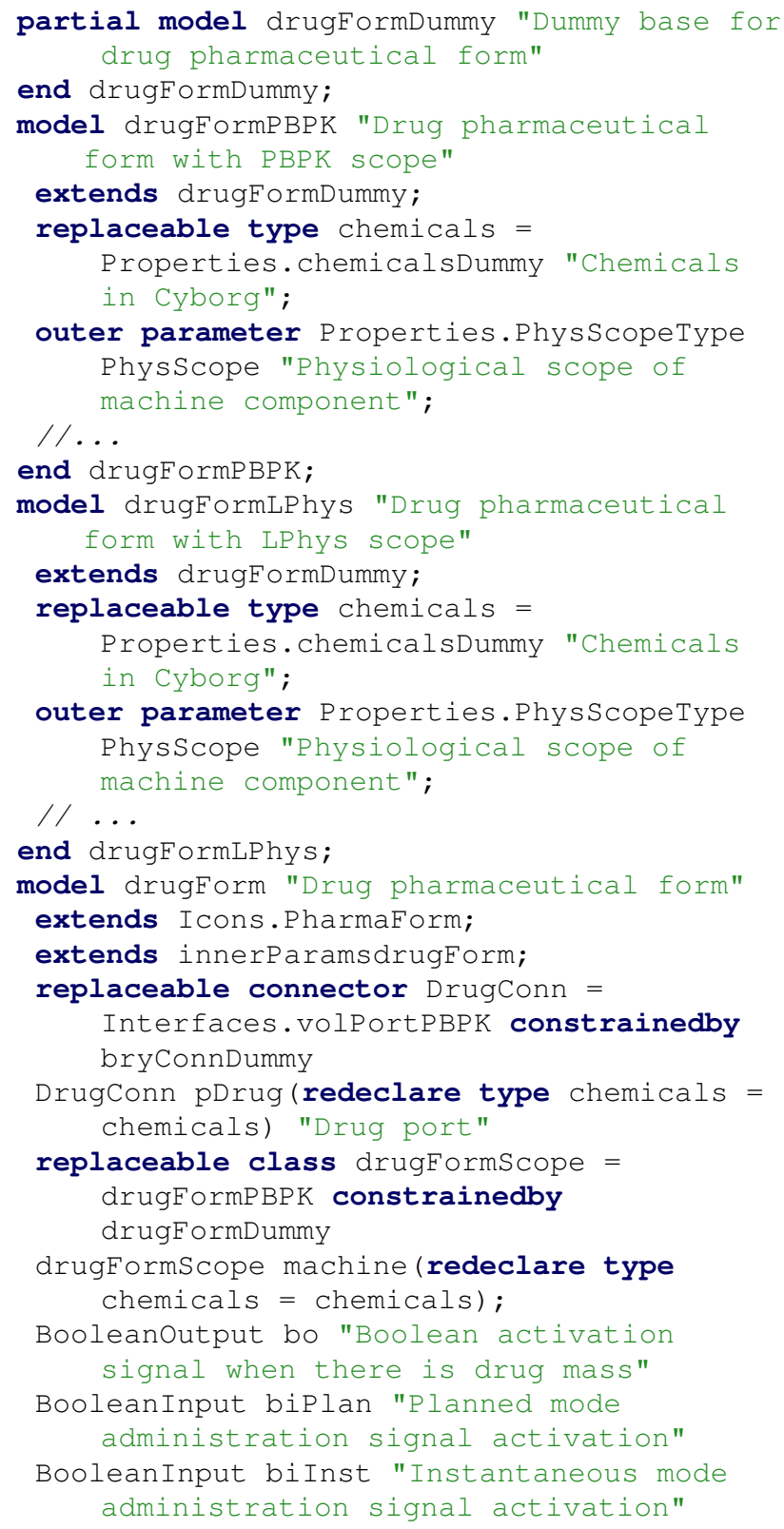




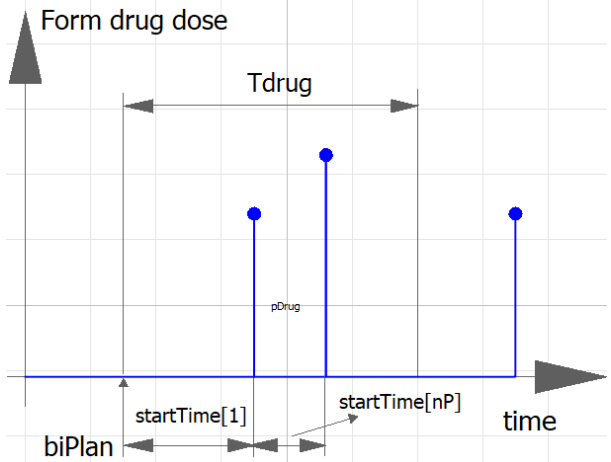

Figure 4. Main planned administration parameters in the drug form. Tdrug is the temporal period, startTime is the starting time of each drug administration in Tdrug, and the number of administrations is $\mathrm{nP}$.

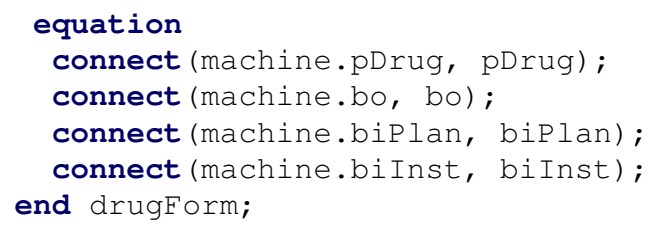

The Drug form may perform both a planned and instantaneous pill administration, controlled by the boolean inputs biPlan and biInst. The Figure 4 shows the simplified diagram that appears in the drug form canvas to clarify the meaning of several planned mode parameters. The temporal area of the pill's surface is modelled according to its geometry. The area is considered by the connected liberation locus (physiology) as a function of the underlying physicochemical mechanism (Berrozpe, Lanao, and Guitart 2013, Ch. 19).

Other features of the CybSim design includes the execution of physiological algorithms to calculate partition ratios, in-vitro in-vivo extrapolation, and different types of physiological scaling as functions of the BioData package.

\subsection{Mechanisms - Physiological layers}

This Section addresses the key aspects of the CybSim design that supports the mechanisms - physiological multilayer feature. I have selected a flow limited tissue (FLT) (Berrozpe, Lanao, and Guitart 2013, Ch. 13) under the PBPK modeling approach (CybSim physiological scope) as a basic tissue that facilitates the description. In this respect, the aim of the study is not to present the full equations and assumptions of a full PBPK either LPhys model.

The Figure 5 describes the Modelica classes associated with the definition of the cited FLT component, called sgnBryFlt, which is shown in Figure 6. Besides the arterial and venous blood paths that connect to the tissue spatial region, the chemical compounds may be transferred in this tissue through a physiological barrier connected to the boundary port. The main variables related to the FLT dynamics may connected via the signal connections to datadriven models, as describen in the previous Section.
The FLT classes structure presented in Figure 5 was designed to allow the selection of the underlying physical mechanisms during model building. The eligible mechanisms for a PBPK component are organized according to functional types in the sgnGeneralPBPK partial component (Figure 5). A similar partial component, called generalPBPK is used if the physiological component does not require connectors to the Signals layer.

In agreement with the Figure 5, the fundamental structure of a PBPK FLT component appears in Listing 4. In this example, the mechanisms are inherited through the partial class generalPBPK. The equations section that appears commented in Listing 4 include very basic equations that complete the definition of the $f l t$ connector variables.

Listing 4. Flow limited tissue code structure

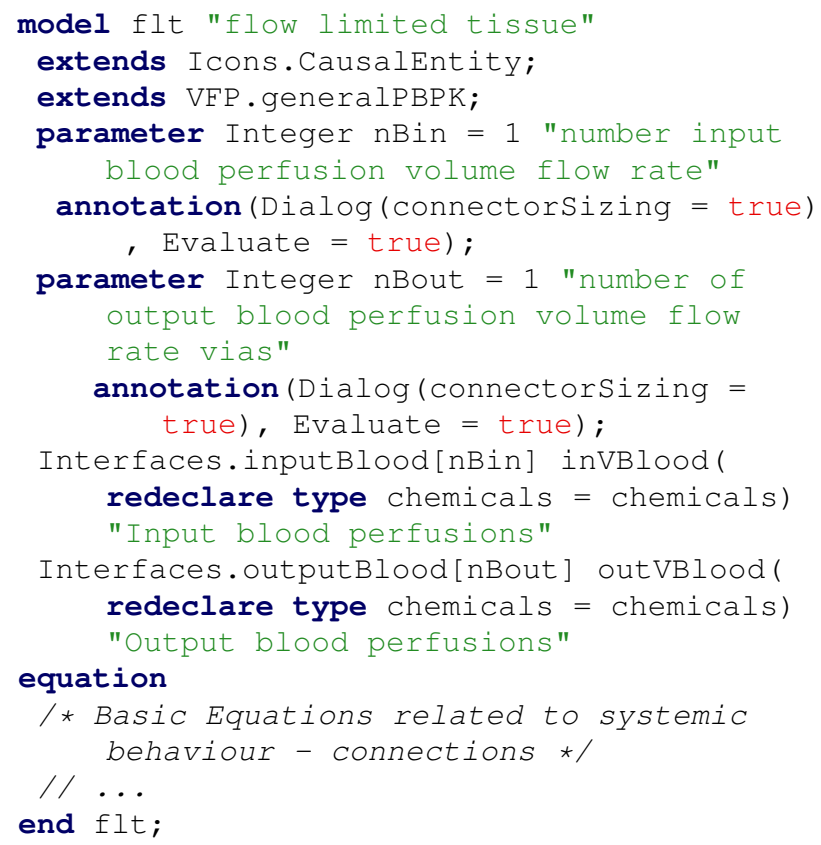

As pointed in Figure 5, the physiological dynamics defined by the partial class sgnGeneralPBPK (and generalPBPK) is obtained through the inheritance of mechanisms organized by functional types. The code structure of generalPBPK partial class is shown in Listing 5. In opposition to sgnGeneralPBPK that inherits VolRegionSgnPBPK, the component sgnGeneralPBPK inherits directly innerWholeVarsPBPK since it has not conditional interfaces to the Signals layer (see right column of Figure 5). A deeper description of this component exceeds the scope of the paper.

Listing 5. general PBPK dynamics of a volumetric region

partial model generalPBPK "General whole mechanism for volumetric regions with PBPK scope"

extends Interfaces.innerWholeVarsPBPK; extends PP.MassBalance.MasterPBPK;

extends PP.Elimination.MasterPBPK; extends PP.PhaseDistribution. MasterPBPK; extends PP.ChemicalActivity.MasterPBPK; extends $\mathrm{PP}$.MolecularBinding.MasterPBPK; equation // Implicit relationship among variables 


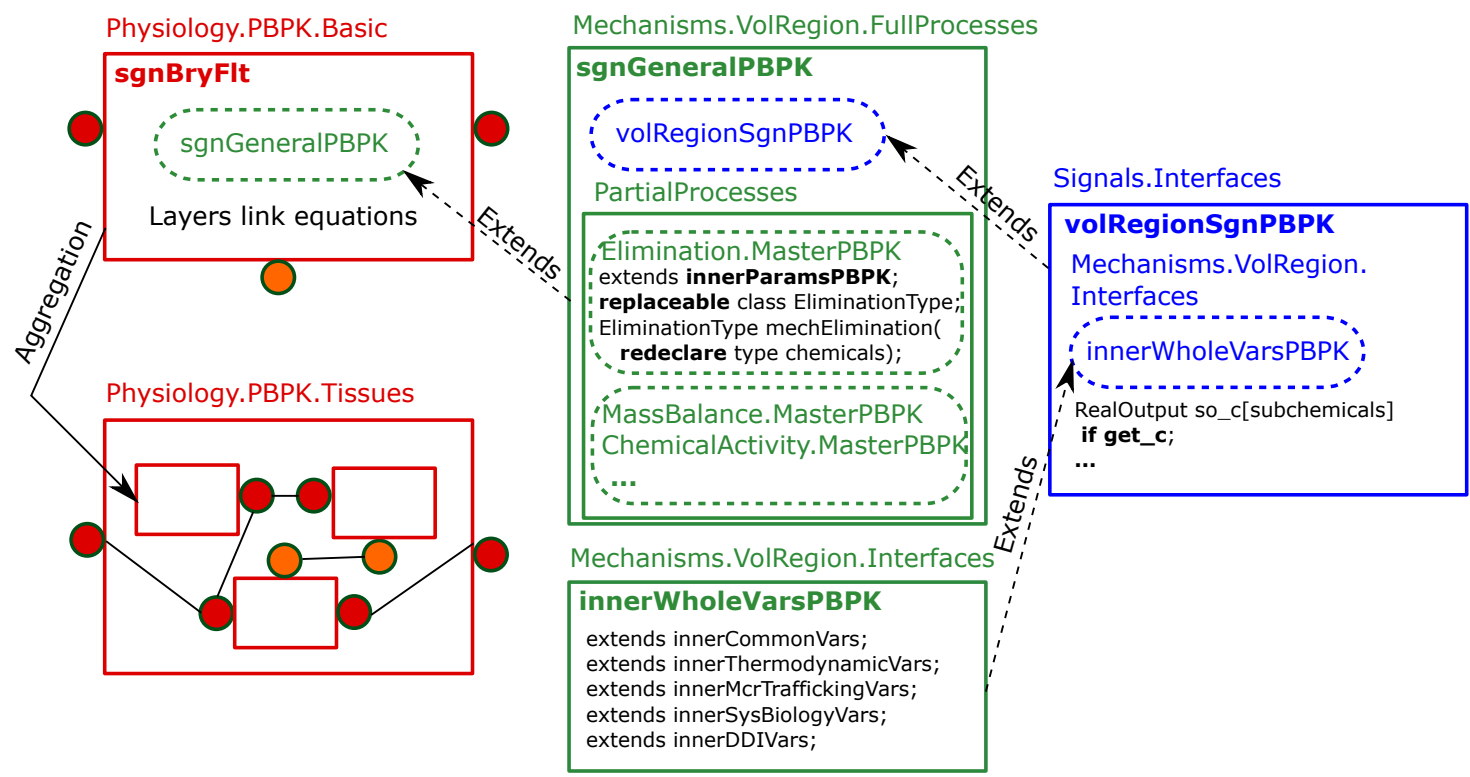

Figure 5. Simplified classes structure of the sgnBryFlt component (left blocks) in the Physiology layer, which inherits the mechanisms sgnGeneralPBPK where it is defined the component dynamics. The sgnGeneralPBPK partial component is defined through a set of replaceable classes. Each one describes a type of eligible behaviour (metabolism, elimination, binding, etc.) related to a spatial volumetric region (partial processes, middle blocks). They are defined through equations that govern the variables of the spatial region, declared as inner variables in the innerWholeVarsPBPK partial component. Partial mechanisms work with the associated outer variables. The sgnGeneralPBPK full mechanism inherits volRegionSgnPBPK (right block) that in turn inherits innerWholeVarsPBPK and declare some conditional structures that simplifies the connection of metrics and other functional models of the Signals layer to the sgnBryflt component.

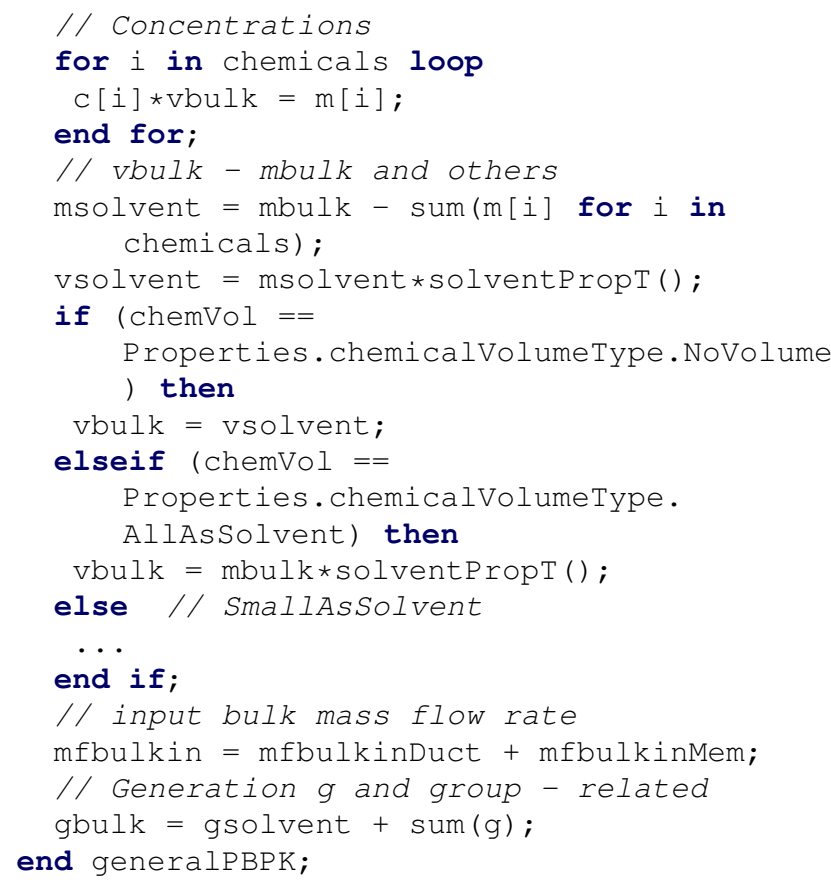

The eligible physical mechanism (see Figure 5) is called MasterPBPK and it is organized in packages according to the behaviour type. The Listing 6 shows the code of MasterPBPK for the elimination behaviour. The class dummyPBPK is defined to control the eligible elimination mechanisms.

Listing 6. Master model of the elimination dynamics in a volumetric region

partial model MasterPBPK "Master model for

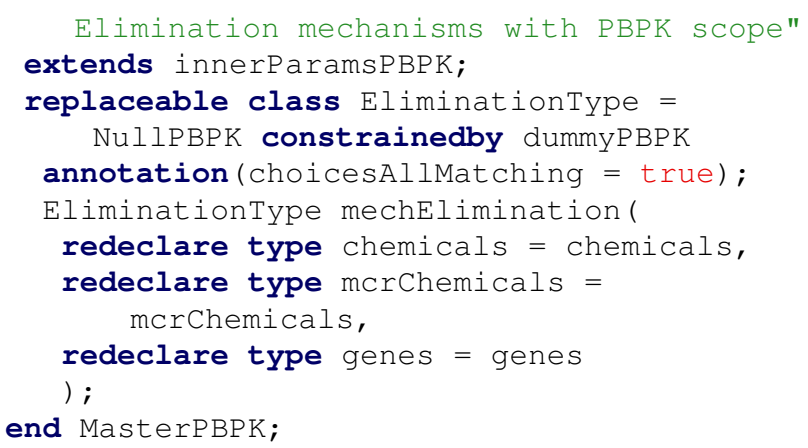

The default elimination mechanism, Elimination.NullPBPK is the null elimination mechanism. Any volumetric region with this elimination mechanisms does not eliminate chemical compounds. The linear plus Michaelis - Menten (saturable) elimination is a well-known mechanism that describes this type of behaviour in Pharmacokinetics. The removal of any chemical $i$ according this one is as follows:

$$
e_{i}=\left(K_{e, i}+\frac{V_{e m, i}}{K_{e m, i}+C_{i}}\right) \cdot C_{i}
$$

in which the concentration $C_{i}$ is equal to the unbound significant phase (tissue) concentration $c_{u, i}$ if the mechanism's parameter significantPhase is true, and to the unbound nonsignificant phase (venous) concentration, $c_{n u, i}$ otherwise. In homogeneous volumetric regions both concentrations are the same.

Assuming that significantPhase is true, the Equation 1 may also be written as follows:

$$
e_{i}=C l_{i} \cdot c_{u, i}
$$

in which the term $C l_{i}$ is the intrinsic clearance of chemical $i$ in 
the region and phase considered, given as:

$$
C l_{i}=K_{e, i}+\frac{V_{e m, i}}{K_{e m, i}+c_{u, i}}
$$

The variables $c_{u, i}$ must be substituted by $c_{n u, i}$ in equations (2) and (3) if the elimination occurs in the non-significant phase. A detailed description of the tissue intrinsic clearance for a well-stirred region is shown in (Pang and Malcolm Rowland 1977). The elimination mechanism related to Equation 2 is implemented in Listing 7.

Listing 7. Saturable elimination dynamics in volumetric region

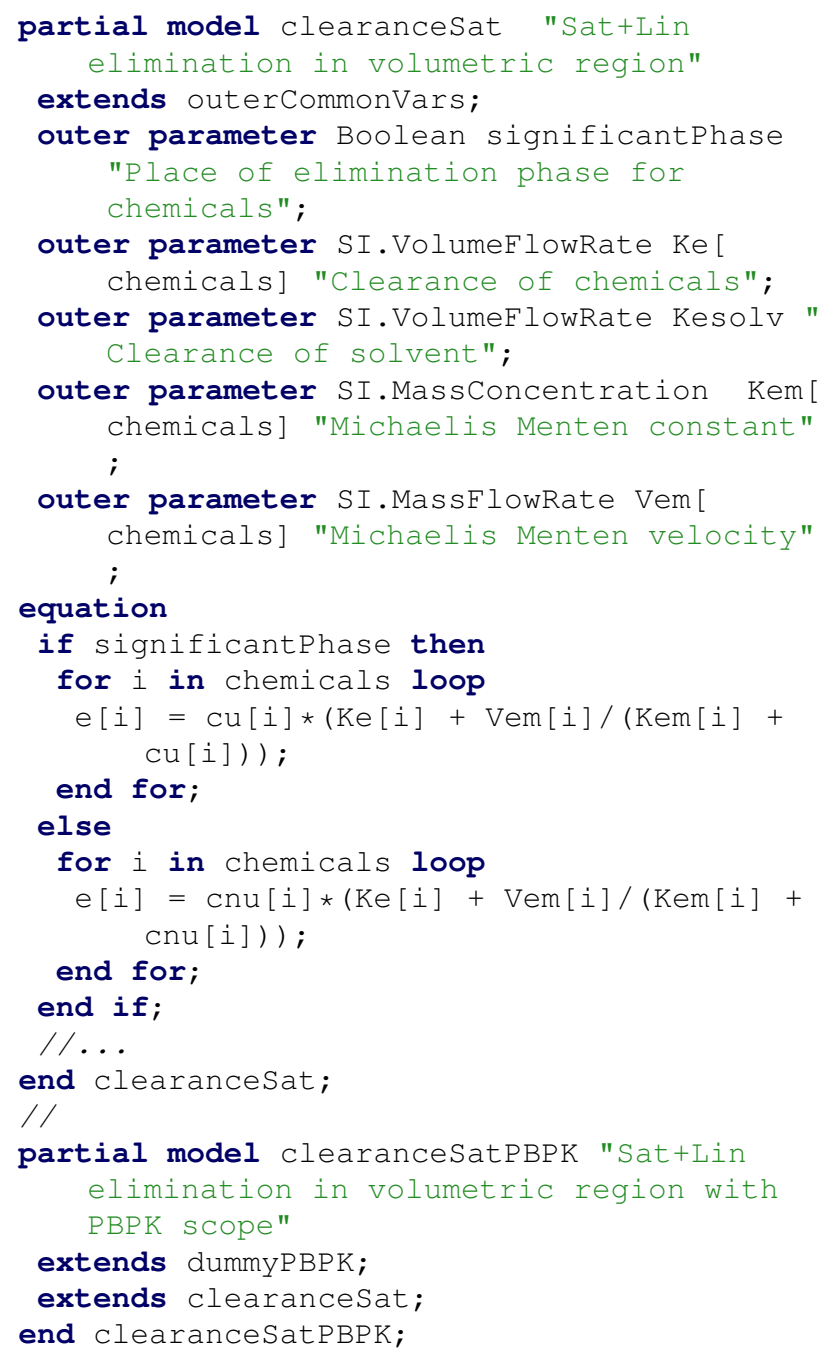

The variables that define the dynamics in a volumetric region are defined in the partial class outerCommonVars, which is
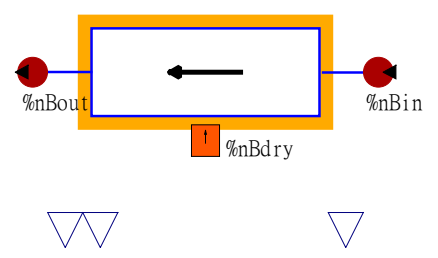

Figure 6. Flow limited tissue (flt) PBPK component with blood ports (circles left-right), boundary port (rectangle below) and signal connections (triangles) sgnBryFlt. accesible to any single mechanism. The associated inner parameters are defined in the Master component, inherited from innerParamsPBPK (Listing 6 and Figure 5). A deeper description of these outer-inner definitions exceeds the scope of the paper.

The mechanism that defines the elimination behaviour is selected during the procedure of building the model, through the declaration of the sgnBryflt physiological component, as seen in the Listing 8 for the central instance. As seen, the value of the $K_{e, i}$ linear parameter is modified from its default value, using a 3 -vectorial expression, what indicates that three chemical compounds are defined.

Listing 8. Declaration of a FLT instance (central) in a PBPK model

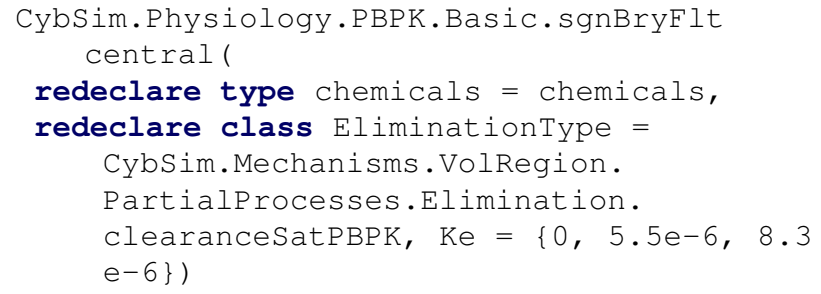

\section{Study case}

Figure 7 shows the diagram of the model defined in the Methods Section. A detailed description of this one appears in (MangasSanjuan et al. 2018), whereas the comparative analysis of the PhysPK vs NONMEM implementations may be seen in (ReigLopez et al. 2020). The building of this model was performed using the GUI of openModelica 1.14.1 (diagram view), although the selection of non-default mechanisms for the physiological components were completed in the Modelica code view of the model, because openModelica 1.14 does not include this graphical function.

The model has been parameterized for a low dose of PD (100 $\mathrm{mg}$ ) administered with two intakes of $50 \mathrm{mg}$ separated $12 \mathrm{~h}$, for a high absorption rate constant in the gut (drug of class II of Biopharmaceutics Classification System), saturable metabolism in gut and liver, and a reference value of the dissolution rate constant (quality level).

The parameter values agree with those used in the first case of (Mangas-Sanjuan et al. 2018, Fig. 2), excepting the division of the $100 \mathrm{mg}$ PD dose in two separated $50 \mathrm{mg}$ PD doses, which is applied now in agreement with the second step pointed in Methods to analyze a more complex scenario, after validating the accuracy of the model against the results of (Mangas-Sanjuan et al. 2018) and (Reig-Lopez et al. 2020).

The final objective of this case study is to demonstrate the feasibility of CybSim to select and change the underlying mechanisms of the model physiological instances during the building process. With this goal, after simulating the model with the liver mechanisms defined in (Mangas-Sanjuan et al. 2018) (first simulation), a linear molecular binding mechanism is added to the liver and a new simulation is executed (second simulation).

The Listing 9 shows the structure of the Modelica code of the semiphysiological model, including the definition of the Liver tissue. The chemicals enumeration is set through the three required chemicals compounds, PD, PM, and SM. The mechanism associated with the chemical activity in the liver is redeclared as a saturable metabolism (MichaelisMentenPBPK), during the initial model building. The boolean inputs bi Inst 


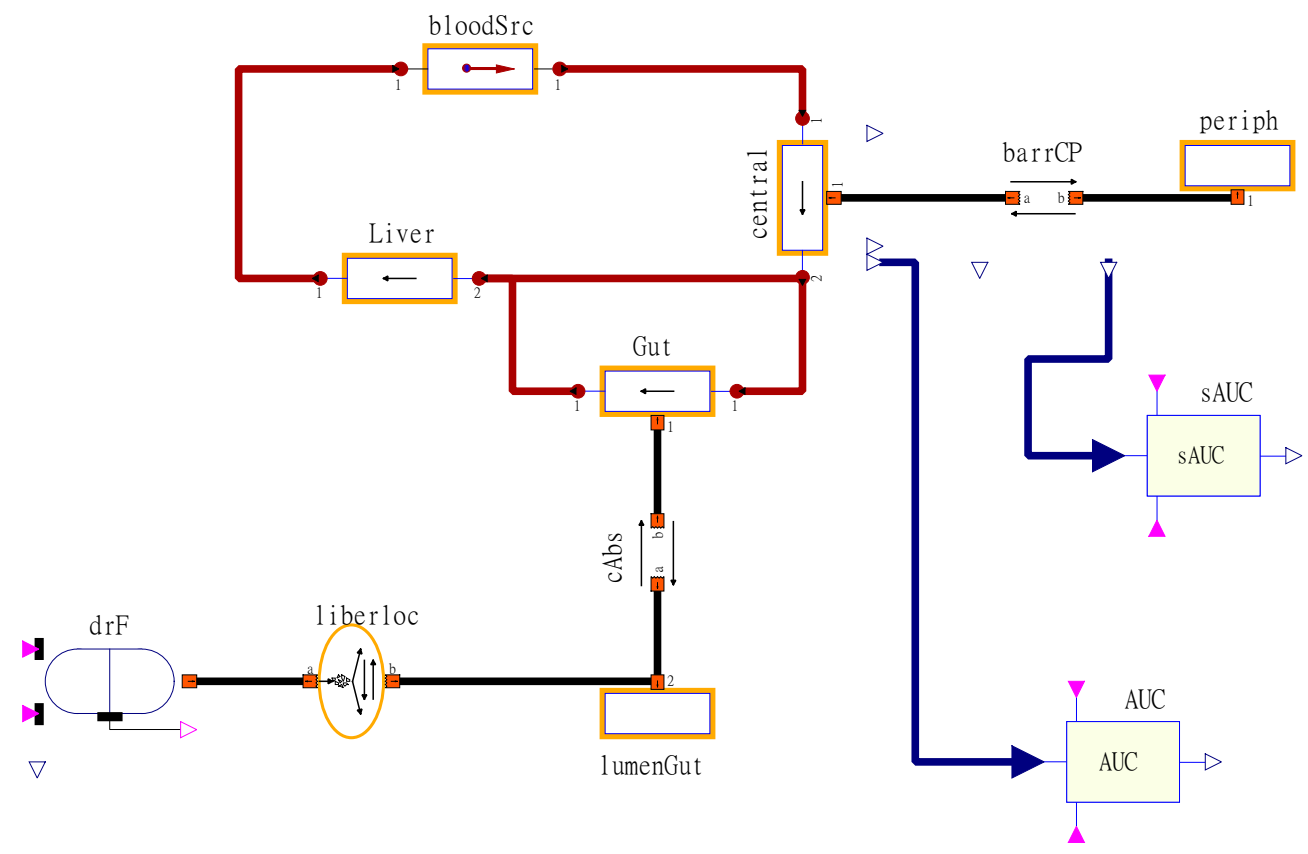

Figure 7. Semiphysiological PBPK model published in (Mangas-Sanjuan et al. 2018; Reig-Lopez et al. 2020) that includes a drug form (drF) from the machine layer, and two PK metric blocks (AUC and sAUC) from the signals layer.

and biPlan define a true planned and false instantaneous administration mode for the drug form, as wished.

The execution of the model for a temporal window of 24 hours gives the plasmatic concentrations of PD, PM and SM under the defined scenario (first simulation).

Listing 9. Main code structure and liver definition of semiphysiological model

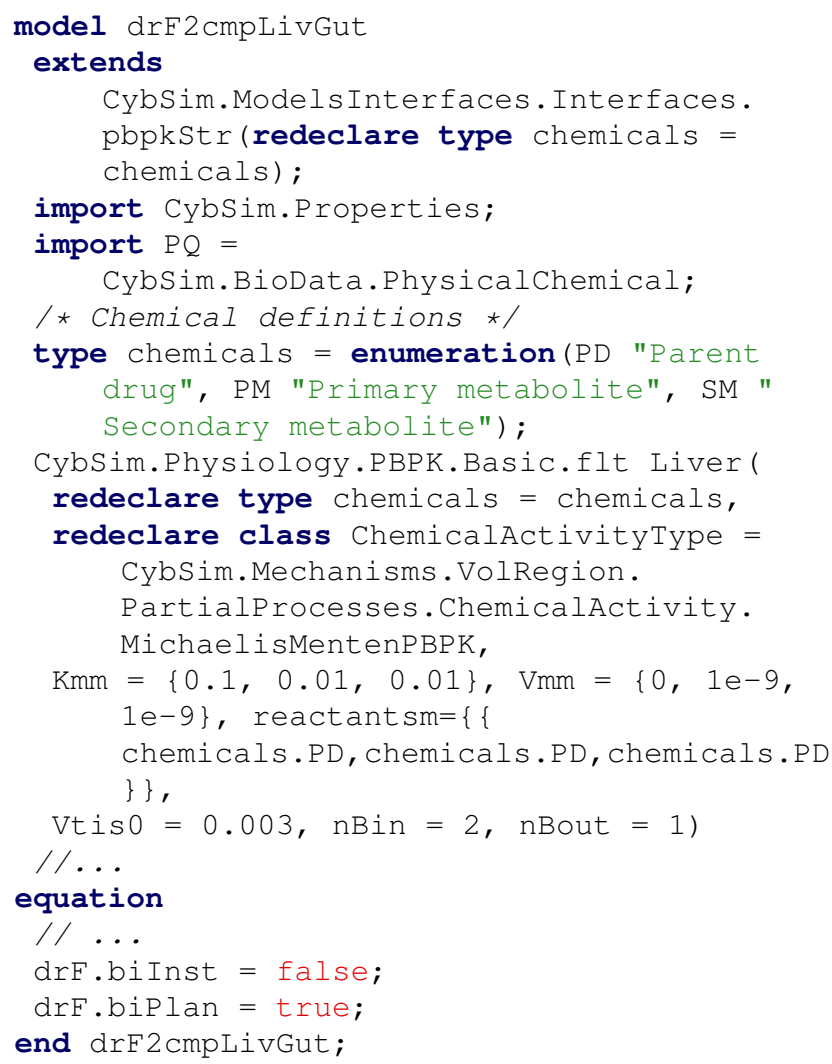

The Listing 10 shows how a linear molecular binding is applied to substitute the default (null) molecular binding of the liver instance of the semiphysiological model.

The linear molecular binding mechanism modifies the amount of free drug according to the unbound fraction drug values $f_{u, i}$. These fractions are defined in the liver declaration as $5 \%$ (0.05) for the PD, and $100 \%$ (1) for the metabolites. That is, the $\mathrm{PD}$ is the unique compound that is bound to a macromolecule, in such a way that only $5 \%$ is free. This is a very common situation in physiological models.

Listing 10. Definition of the FLT liver instance with linear binding

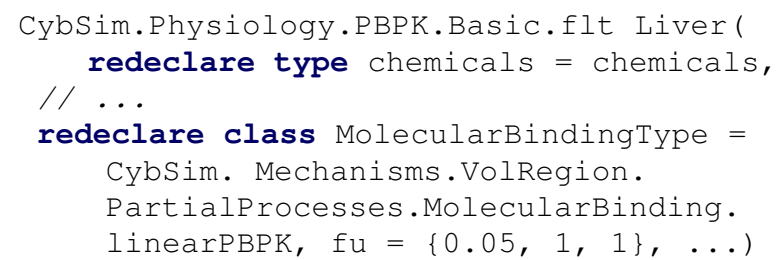

The execution of the model for the same temporal window of 24 hours gives the plasmatic concentrations of PD, PM and SM in the second simulation.

\section{Simulation results and discussion}

Figure 8 shows the plasmatic (central) concentrations of the parent drug and their metabolites during the first 24 hours, starting with the first PD dose (50 mg).

The temporal distribution of the chemical compound was accurately validated both for an unique dose of PD equal to 100 mg (Mangas-Sanjuan et al. 2018, Fig. 2) and for two sequential doses of PD equal to $50 \mathrm{mg}$. A detailed analysis of this testing phase exceeds the scope of this paper that is focused to demonstrate the feasibility of the CybSim design to support the mechanisms - physiology architecture. 


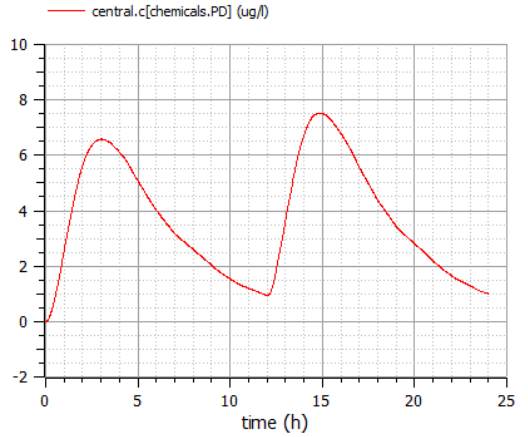

(a) Parent Drug.

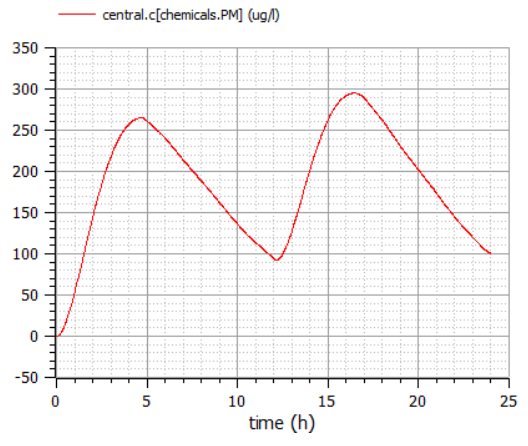

(b) Principal metabolite.

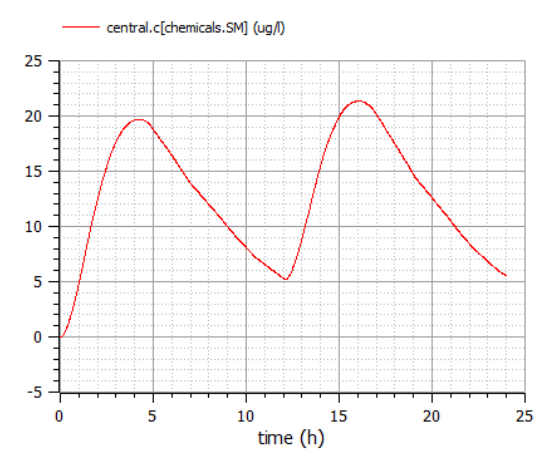

(c) Secondary metabolite.

Figure 8. Plasma (central) concentrations for a low dose scheme $(50+50 \mathrm{mg})$ without liver molecular binding.

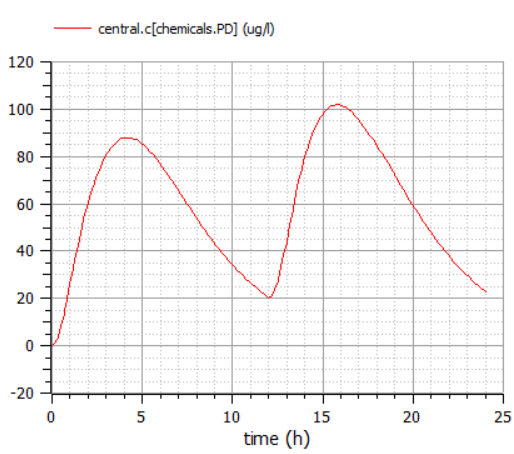

(a) Parent Drug.

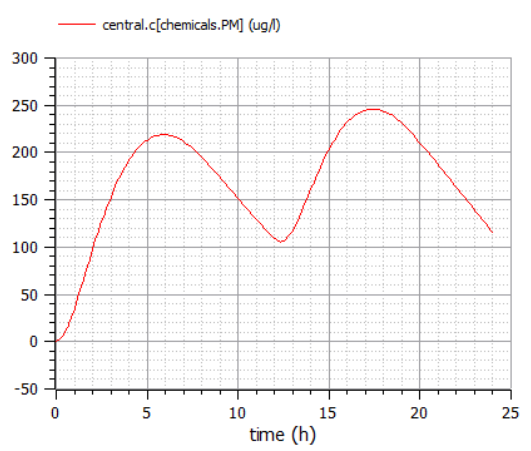

(b) Principal metabolite.

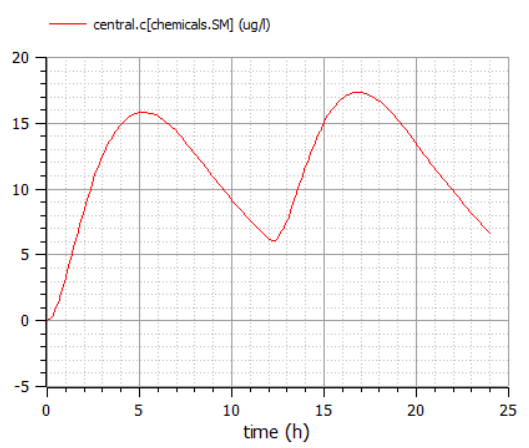

(c) Secondary metabolite.

Figure 9. Plasma (central) concentrations for a low dose scheme $(50+50 \mathrm{mg})$ with liver molecular binding for $\mathrm{PD}\left(f_{u}=0.05\right)$.

The Figure 9 shows the plasmatic concentrations of PD and their metabolites during the first 24 hours for the second simulation. As expected, the PD concentration increases, whereas PM and SM concentrations decrease, with respect to the first simulation, due to the reduction of liver metabolism because of the smaller amount of free PD.

The Area Under the Curve (AUC) blocks, which are connected to the concentrations (all the chemicals) inside the central compartment (AUC block) and to the total mass flow rate from central to peripheral compartment (sAUC or single AUC), are not presented here. However, they were used to evaluate the parent drug and metabolites AUC in the target central compartment and to calculate the total net amount of mass between central and peripheral compartment during the first 24 horas. They were used also to demonstrate the capabilities of CybSim related to the Signals layer.

Although a detailed comparison between CybSim and PhysPK exceeds the scope of this paper, CybSim overcomes several important limitations of PhysPK due to the lack of classes' redeclaration, inner/outer structures, packages organization, and causality of connectors. These language characteristics are the basis of the mechanisms - physiology multilayer, the multiscope feature, the free definition of chemical compounds inside the physiological instances, and many of the capabilities of the signals layer.

\section{Summary}

This study has presented a novel Modeling and Simulation software system for the biosciences field, CybSim, that gives a framework compliant with current methodologies and specific solutions required in particular areas like pharmacology, precision medicine and toxicology. CybSim tries to overpass some detected lacks related to model reusing and sharing in current Modeling and Simulation software systems.

The outcomes demonstrate the feasibility of CybSim to facilitate the choice of the mechanisms underlying the physiological entities in the process of model building. To the best of my knowledge, this is the first biosimulation system that fulfils that feature at the same time that offers modeling multiscope, free definition of chemicals, multilevel modeling, metabolic networks based on the systems biology approach, machines integration and support for data-drive modeling.

Future works will be developed to evaluate those features, and to complete the implementation of other packages related to optimization (applied to population estimation and dosage personalization), and biodata (algorithms for in-vitro in-vivo correlation, allometric scaling, and methods for computation of physiological properties).

This is a first paper concerning the preliminary version 0.2 of the CybSim biosimulation system. More advances will be performed and published shortly, including the evolution to the available openModelica 1.18 that should give better solutions to some relevant planned features. CybSim will be deployed with availability for download under open source license, after reaching the required minimal functionality.

\section{References}

Azer, Karim et al. (2021). "History and Future Perspectives on the Discipline of Quantitative Systems Pharmacology Modeling and Its Applications". In: Frontiers in Physiology 12. 
Berrozpe, José Doménech, José Martinez Lanao, and Concepción Peraire Guitart (2013). Tratado general de Biofarmacia y Farmacocinética. Volumen II [General treatise of biopharmacy and pharmacodynamics. Vol II]. Madrid, Spain: Editorial Síntesis.

Bizzotto, R. et al. (2017). "PharmML in Action: an Interoperable Language for Modeling and Simulation". In: CPT Pharmacometrics Syst Pharmacol 6.10, pp. 651-665.

Bloomingdale, P. et al. (2017). "Quantitative systems toxicology”. In: Curr Opin Toxicol 4, pp. 79-87.

Bonate, Peter L. (2011). Pharmacokinetic-Pharmacodynamic Modeling and Simulation. Second Edition. Springer, p. 618. ISBN: 9781441994844.

Darwich, A. S. et al. (2017). "Why has model-informed precision dosing not yet become common clinical reality? lessons from the past and a roadmap for the future". In: Clin Pharmacol Ther 101.5, pp. 646-656.

Empresarios Agrupados (2019). User Manual EcosimPro 6.0. Report.

Fritzson, Peter (2015). Principles of Object-Oriented Modeling and Simulation with Modelica 3.3. second edition. USA: Wiley, p. 1250. ISBN: 9781-118-859124.

GastroPlus (2021). GastroPlus. Available online: www.simulations-plus.com/software/gastroplus (accessed on 10 May 2021). Web Page.

Gonzalez-Garcia, Ignacio et al. (2017). "Comparison of FO FOCE population parameter estimation methods in PhysPK 2.0 against NONMEM 7.3”. In: PAGE 2017. Abstracts of the Annual Meeting of the Population Approach Group in Europe, pp. 1-2.

Hester, R. L. et al. (2011). "HumMod: A Modeling Environment for the Simulation of Integrative Human Physiology". In: Front Physiol 2, p. 12.

Hunter, Peter J. (2006). "Modeling Human Physiology: The IUPS/EMBS Physiome Project". In: Proceedings of the IEEE 94, pp. 678-691.

Hunter, Peter J. and Marco Viceconti (2009). "The VPHPhysiome Project: Standards and Tools for Multiscale Modeling in Clinical Applications". In: IEEE Reviews in Biomedical Engineering 2, pp. 40-53.

Ježek, Filip et al. (2017). "Lumped models of the cardiovascular system of various complexity". In: Biocybernetics and Biomedical Engineering 37.4, pp. 666-678.

Jones, Hannah M, Iain B Gardner, and Kenny J Watson (2009). "Modelling and PBPK simulation in drug discovery." In: The AAPS journal 11, pp. 155-166.

Keating, S. M. et al. (2020). "SBML Level 3: an extensible format for the exchange and reuse of biological models". In: Mol Syst Biol 16.8, e9110.

Maharao, N. et al. (2020). "Entering the era of computationally driven drug development". In: Drug Metab Rev 52.2, pp. 283-298.

Mangas-Sanjuan, V. et al. (2018). "Computer simulations for bioequivalence trials: Selection of analyte in BCS class II and IV drugs with first-pass metabolism, two metabolic pathways and intestinal efflux transporter". In: Eur J Pharm Sci 117, pp. 193-203.

Mateják, M. et al. (2014). "Physiolibrary - Modelica library for physiology". In: 10th International Modelica conference.

Modelica Association (2017-04). Modelica - A Unified ObjectOriented Language for Systems Modeling. Language Specification Version 3.4. Tech. rep. Linköping: Modelica As- sociation. URL: https : / / www . modelica . org / documents / ModelicaSpec34.pdf.

NONMEM (2021). NONMEM. Available online: www.iconplc.com/innovation/nonmem/ (accessed on 17 Jul 2021). Web Page.

Open-Systems-Pharmacology (2021). Open-SystemsPharmacology. Available online: www.open-systemspharmacology.org (accessed on 10 May 2021). Web Page.

Paini, A. et al. (2019). "Next generation physiologically based kinetic (NG-PBK) models in support of regulatory decision making". In: Comput Toxicol 9, pp. 61-72.

Pang, K. Sandy and Malcolm Rowland (1977). "Hepatic Clearance of Drugs. I. Theoretical Considerations of a "WellStirred" Model and a "Parallel Tube" Model. Influence of Hepatic Blood Flow, Plasma and Blood Cell Binding, and the Hepatocellular Enzymatic Activity on Hepatic Drug Clearance". In: Journal of Pharmacokinetics and Biopharmaceutics 5.6.

Polasek, T. M., S. Shakib, and A. Rostami-Hodjegan (2019). "Precision medicine technology hype or reality? The example of computer-guided dosing". In: Fl000Res 8, p. 1709.

Prado-Velasco, Manuel (2016). "Bridging the gap between open and specialized modelling tools in PBPK/PK/PD with PhysP$\mathrm{K} /$ EcosimPro modelling system: PBPK model of methotrexate and 6-mercaptopurine in humans with focus in reusability and multilevel modelling features". In: PAGE. Abstracts of the Annual Meeting of the Population Approach Group in Europe, pp. 1-2.

Prado-Velasco, Manuel, Alberto Borobia, and Antonio CarcasSansuan (2020). "Predictive engines based on pharmacokinetics modelling for tacrolimus personalized dosage in paediatric renal transplant patients". In: Scientific Reports 10.1, p. 7542 .

Reig-Lopez, J. et al. (2020). “A Multilevel Object-Oriented Modelling Methodology for Physiologically-Based Pharmacokinetics (PBPK): Evaluation with a Semi-Mechanistic Pharmacokinetic Model". In: Computer Methods and Programs in Biomedicine 189, pp. 1-11.

Roa, Laura and Manuel Prado (2006). "Simulation Languages". In: Wiley Encyclopedia of Biomedical Engineering. Ed. by Metin Akay. John Wiley and Sons, Inc., pp. 3186-3198. ISBN: 978-0-471-24967-2.

Rowland, M, Lj Lesko, and A Rostami-Hodjegan (2015). "Physiologically Based Pharmacokinetics Is Impacting Drug Development and Regulatory Decision Making". In: CPT: Pharmacometrics \& Systems Pharmacology 4, pp. 313-315.

Sauro, Herbert M. et al. (2004). "Next Generation Simulation Tools: The Systems Biology Workbench and BioSPICE Integration". In: OMICS: A Journal of Integrative Biology 7.4.

Shepard, T et al. (2015). "Physiologically Based Models in Regulatory Submissions : Output From the ABPI / MHRA Forum on Physiologically Based Modeling and Simulation". In: pp. 1-5.

SimCyp (2021). SimCyp. Available online: www.certara.com/software/simcyp-pbpk (accessed on 10 May 2021). Web Page. 